
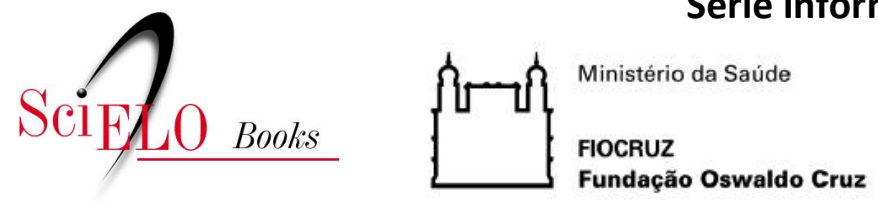

OBSERVATÓRIO COVID-19

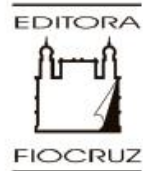

Parte III - Covid-19 e Fiocruz

\title{
22. A estratégia de redes estruturantes na cooperação internacional da Fiocruz: América Latina e CPLP
}

\author{
Sebastián Tobar \\ Felix Júlio Rosenberg \\ Augusto Paulo José da Silva \\ Rosa Maria Pinheiro Souza \\ Pedro Burger \\ Beatriz Nascimento \\ Felippe Amarante \\ Helifrancis Condé Groppo Ruela \\ Ana Beatriz de Noronha \\ Luciana Frederico Milagres \\ João Aprígio
}

\section{SciELO Books / SciELO Livros / SciELO Libros}

TOBAR, S., ROSENBERG, F.J., SILVA, A.P.J., SOUZA, R.S.P., BURGER, P., NASCIMENTO, B., AMARANTE, F., RUELA, H.C.G., NORONHA, A.B., MILAGRES, L.F., and APRÍGIO, J. A estratégia de redes estruturantes na cooperação internacional da Fiocruz: América Latina e CPLP. In: BUSS, P.M., and FONSECA, L.E. eds. Diplomacia da saúde e Covid-19: reflexões a meio caminho [online]. Rio de Janeiro: Observatório Covid 19 Fiocruz; Editora FIOCRUZ, 2020, pp. 345-360. Informação para ação na Covid-19 series. ISBN: 978-65-5708-029-0. https://doi.org/10.7476/9786557080290.0023.

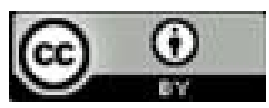

All the contents of this work, except where otherwise noted, is licensed under a Creative Commons Attribution $\underline{4.0 \text { International license. }}$

Todo o conteúdo deste trabalho, exceto quando houver ressalva, é publicado sob a licença Creative Commons Atribição 4.0. 


\section{A Estratégia de Redes Estruturantes na Cooperação Internacional da Fiocruz} América Latina e CPLP

Sebastián Tobar, Felix Júlio Rosenberg, Augusto Paulo José da Silva, Rosa Maria Pinheiro Souza, Pedro Burger, Beatriz Nascimento,

Felippe Amarante, Helifrancis Condé Groppo Ruela, Rosa Maria Pinheiro Souza, Ana Beatriz de Noronha, Luciana Frederico Milagres e João Aprígio

Em II de março de 2020, o diretor-geral da Organização Mundial da Saúde (OMS) L declarou a Covid- 19 como uma pandemia. Cinco meses depois, a enfermidade já atinge mais de 20 milhões de pessoas em todo o planeta, causando mais de 700 mil mortes, metade das quais acontecem na América Latina.

$\mathrm{Na}$ África, ainda que o número de casos registrados represente apenas $5 \%$ do total global, até agosto foram confirmados mais de um milhão de adoecimentos, com quase 25 mil mortes. Nos países africanos que integram a Comunidade dos Países de Língua Portuguesa (CPLP) - Angola, Cabo Verde, Guiné-Bissau, Guiné Equatorial, Moçambique e São Tomé e Príncipe -, o total gira em torno de 15 mil casos registrados, com pouco mais de 250 óbitos, o que representa aproximadamente 1,4\% dos casos e I, I\% das mortes ocorridas no continente.

Nesse sentido, a Covid- 19 confirma-se como uma questão de saúde global, definida como

problemas de saúde que transcendem as fronteiras nacionais e os governos e que requerem intervenções nas forças e fluxos globais que determinam a saúde das pessoas. Requer novas formas de governança em nível nacional e internacional, as quais procuram incluir uma ampla gama de atores. (Kickbusch \& Berger, 20I0)

No contexto pandêmico, o trabalho em rede constitui uma grande oportunidade de atuar compartilhando esforços e promovendo a construção de capacidades por meio da cooperação para o desenvolvimento da saúde. 


\section{ASPECTOS CONCEITUAIS}

O conceito de redes tem sido utilizado há algum tempo na área da saúde, com diversos significados. Em geral, o termo pode ser usado em situações onde é possível identificar interações ou trocas entre diferentes membros, os nós da rede, para enfrentar desafios comuns. As redes podem ser de profissionais, de pessoas ou de instituições. Mesmo assim, em última análise, é por meio das pessoas que as instituições que compõem uma rede são conectadas.

No caso da Fundação Oswaldo Cruz (Fiocruz), trata-se de conectar pessoas ou instituições que não se constituam "em si", mas como "redes para" ou "redes meio" (Rovere, 1998), que procuram dar uma resposta melhor a determinado problema de saúde pública ou ao sistema de saúde, provendo uma ação coletiva.

O trabalho em rede permite a articulação de diferentes atores pertencentes a instituições que se agrupam voluntariamente e possibilita a troca e a conjugação de esforços, experiências e conhecimentos para o alcance de objetivos ou de solução para problemas comuns. Nesse sentido, a noção de rede está intimamente relacionada às relações ou vínculos entre os atores que pertencem às instituições.

A ligação entre membros da rede tem o propósito de que estes possam desenvolver atividades comuns, procurar atingir determinados objetivos ou resolver problemas, o que os faz constituírem uma identidade comum. Dessa forma, o interesse comum entre pessoas ou instituições é essencial para "envolver" os participantes da rede.

O trabalho em rede é caracterizado pela adaptabilidade dos membros das redes e pela flexibilidade, abertura, horizontalidade, fluidez e espontaneidade das relações estabelecidas entre eles.

As pessoas ou instituições "enredam" ou constroem redes a partir de esforços conjuntos ou cooperam com base no valor da heterogeneidade entre eles para o benefício conjunto. Uma rede envolve conectar pessoas e instituições dentro de uma heterogeneidade. Então, construir redes envolve assumir a heterogeneidade, permitindonos imaginar uma heterogeneidade organizada (Rovere, 1998).

\section{A PANDEMIA COMO UM DESAFIO COMUM}

Em um cenário de maior vulnerabilidade como o atual, no qual a Covid- 19 traspassa as fronteiras nacionais, globalizando seus riscos sanitários, sociais e econômicos, o trabalho em rede se apresenta como grande oportunidade para a cooperação em saúde. 
A Região das Américas tornou-se o epicentro atual da pandemia de Covid-19. O diretor-geral da OMS tem reiterado que, "apesar de todas as nossas diferenças, somos uma raça humana compartilhando o mesmo planeta e nossa segurança é interdependente - nenhum país estará seguro até que estejamos todos seguros”. Mais que nunca, a pandemia veio mostrar que saúde e bem-estar é um desafio comum a todos em todos os lugares.

A globalização, comumente atrelada ao progresso e ao desenvolvimento, também excluiu grande parte da população mundial que não usufrui seus benefícios. A pandemia escancarou essas desigualdades ao afetar diferentemente grupos populacionais e de países. Mesmo com cenários diferentes, a Covid-19 atingiu praticamente todos os países, tornando-se, portanto, uma questão cujo enfrentamento traz em si um desafio comum, tornando-se também um desafio às abordagens em rede no campo da saúde global, que já vinham se implantando antes da crise.

No início deste século, tivemos importantes exemplos de cooperação em saúde por meio de redes, os quais poderiam servir de modelo na luta contra a nova pandemia do Sars-2Cov: o Conselho de Saúde Sul-Americano da União de Nações Sul-Americanas (Unasul) e o Conselho dos Ministros da Saúde da CPLP. Nesses processos de integração regional - Unasul e CPLP -, a Fiocruz impulsionou uma modalidade de trabalho em rede chamada "cooperação estruturante".

\section{COOPERAÇÃO ESTRUTURANTE: MODELO EM REDE}

Buscando um processo de cooperação mais adequado para os países em desenvolvimento, a Fiocruz promove, desde 2009, uma nova modalidade de cooperação denominada "cooperação estruturante em saúde". Essa modalidade incorpora um planejamento estratégico focado nas realidades nacionais de cada país ou instituição, entendendo-os como parceiros, e por isso descarta a concepção tradicional de doadores e destinatários da cooperação. Essa perspectiva deixa de lado os programas de ajuda verticais (intervenções com foco em doenças ou problemas específicos), adotando um enfoque horizontal, voltado para o desenvolvimento integral dos sistemas de saúde.

Essa nova perspectiva enfatiza o longo prazo e a construção de capacidades nos países por meio do fortalecimento das instituições estruturantes dos sistemas de saúde para que adquiram liderança genuína nos processos nacionais; permite avançar para a saúde universal; busca estabelecer o equilíbrio entre ações específicas voltadas para a resolução de problemas imediatos com geração de conhecimento e desenvolvimento de capacidades institucionais nacionais sustentáveis; incorpora na cooperação em 
saúde a perspectiva dos determinantes sociais da saúde e das ações intersetoriais; e prioriza programas de saúde pública (com foco na população), ao invés de programas e atividades voltados estritamente para indivíduos.

No marco da extinta Unasul e da CPLP, a Fiocruz tem promovido a criação de diversas redes visando a superar as fragilidades dos componentes dominantes da cooperação, as quais se orientam para o desenvolvimento institucional dos sistemas de saúde dos países parceiros. Essas ações buscam conjugar

intervenções específicas de construção de capacidades locais e de geração de conhecimento, promovendo o diálogo entre os atores, de forma a capacitá-los a assumir protagonismo na liderança dos processos do setor saúde e promover a formulação autônoma de uma agenda para o desenvolvimento do setor. (Almeida et al., 20। 0: 28)

Trata-se, portanto, de apoiar o desenvolvimento do sistema de saúde em suas várias dimensões, buscando a concretização de sistemas baseados em direitos, com universalidade, integralidade, qualidade e equidade.

Vários autores veem as redes como potencialmente capazes de construir sujeitos dispostos e aptos a transformar a realidade (Franco, 2006; Souza \& Costa, 20 I 9). O cotidiano das práticas de saúde é caracterizado por permanentes fluxos operacionais, políticos, comunicativos e simbólicos, os quais, incorporados em rede, conferem aos trabalhadores a ideia de pertencimento entre si, entre os trabalhadores e os equipamentos de saúde, e a uma rede maior que opera conectando fluxos com outros computadores e usuários.

O trabalho em rede permite que diferentes grupos de atores sociais e políticos resolvam problemas comuns, constituindo uma estratégia organizacional para compartilhar recursos, práticas e conhecimentos entre as instituições de forma a maximizar a eficácia e eficiência de suas ações.

Com a infeliz suspensão da Unasul, patrocinada pelos governos de Argentina, Brasil, Chile, Colômbia, Chile, Peru e Paraguai, perdeu-se um importante espaço político regional e de saúde na América do Sul, no momento de pandemia de Covid-19, doença que põe em xeque toda a estrutura de governança global, regional e nacional em saúde e que torna extremamente necessária a cooperação mútua.

Por essas razões, a Fiocruz desenvolveu e implementou estratégias para continuar o trabalho em rede, por meio de outros guarda-chuvas institucionais, promovendo o trabalho conjunto. 


\section{REDE DE ESCOLAS E CENTROS FORMADORES EM SAÚDE PÚBLICA DA AMÉRICA LATINA (RESP-AL): UMA NOVA ETAPA DO FUNCIONAMENTO EM REDE}

Com a dissolução da Unasul, a Escola Nacional de Saúde Pública Sergio Arouca (Ensp) e o Centro de Relações Internacionais em Saúde (Cris) da Fiocruz realizaram negociações com a Coordenação do Programa Sub-Regional para a América do Sul da Organização Pan-Americana da Saúde (Opas/OMS), propondo a ressignificação da Resp/ Unasul em uma nova rede.

Em 31 de maio de 2019 foi realizada a primeira reunião sobre a proposta de organização de uma nova rede latino-americana na área da formação em saúde pública, que considerasse os logros alcançados nos anos anteriores pela rede ancorada na Unasul, privilegiando e mantendo as articulações que nasceram entre instituições e pessoas durante os anos de funcionamento da Resp-Unasul. Nesse primeiro momento, contou-se com a participação de instituições da Argentina e do Brasil, Equador, México, Paraguai, Peru e Uruguai. Posteriormente, em dezembro do mesmo ano, foi oficialmente fundada a nova Rede de Escolas e Centros Formadores em Saúde Pública da América Latina (Resp-AL), no contexto do III Colóquio Latino-Americano de Formação em Saúde Pública. Assinaram a ata fundadora instituições de 1 I países. Além dos participantes na primeira reunião, somaram-se instituições da Bolívia, do Chile, da Costa Rica e de Cuba.

$\mathrm{Na}$ criação de uma rede latino-americana consideram-se os desafios da formação de graduação e pós-graduação em saúde pública nos países da região, propondo estratégias de intercâmbio de experiências e saberes e compartilhamento de ações visando ao fortalecimento das estratégias formativas em cada país. Com a proposta de que todos os países-membros formem suas redes nacionais, como já ocorre na Argentina e no Brasil, Chile e Peru, esta iniciativa configura-se como uma "rede de redes", propondo organizar, nos casos apropriados, redes de convocação das instituições nacionais.

Cada uma das instituições que fazem parte da rede está imersa em determinado contexto econômico, político, cultural, epidemiológico, de saúde e na própria história e estrutura dos sistemas de saúde de seus países e, a partir de tal contexto, desenvolve suas funções em pesquisa, desenvolvimento, inovação, educação, bem como em cooperação e ação comunitária.

Um dos aspectos fundamentais na conformação de redes é a comunicação, entendida como crucial para o desenvolvimento de uma cultura de grupo, o que também ajuda a criar um senso comum de realização (Gocan $E$ Laplante, 20I4). Nesse sentido, a Resp-AL tem investido em diversas iniciativas para proporcionar uma comunicação dinâmica entre os diversos países, instituições e profissionais envolvidos, como grupos 
de e-mail e WhatsApp, além da elaboração de um website (http://www.respal.ensp. fiocruz.br/). No contexto da pandemia de Covid- I9, a fluidez no diálogo e as interações constantes têm sido fundamentais, com trocas em tempo real de informações políticas, sociais, epidemiológicas de cada país representado na rede, além de debates e reflexões em torno da conjuntura, dos desafios e das lições aprendidas.

Exemplo concreto da articulação promovida no âmbito da Resp-AL foi a Declaração Conjunta sobre a Pandemia de Covid- I 9, em que seus membros manifestam preocupação com o cenário de ascensão da epidemia em alguns países e com o fato de alguns governos priorizarem a retomada da produção em detrimento da saúde. A declaração enfatiza a importância do distanciamento social combinado com políticas sociais governamentais, como a renda mínima, e da atenção primária como estratégia para uma saúde pública, universal, integral e equânime (Resp-AL, 2020).

Por meio da articulação em rede, as instituições participantes da Resp-AL têm desenvolvido importantes atividades on-line, como debates e seminários sobre temas essenciais no contexto da pandemia de Covid- 19, seja por meio das Redes Nacionais de Formação em Saúde Pública, a exemplo da brasileira RedEscola, da chilena, da peruana e da argentina, seja por meio da própria rede latino-americana. Em reunião virtual, os membros da Resp definiram temas prioritários para o debate conjunto, em uma sequência de webinários a serem coordenados alternadamente. Nesse contexto foi realizado um seminário virtual sobre Vacinas para a Covid- 19 como Bens Globais, em parceria com outras redes como a Rede Internacional de Educação de Técnicos em Saúde (Rets) e a Rede de Institutos da Comunidade de Países de Língua Portuguesa (Rinsp-CPLP).

\section{A REDE DE ESCOLAS NACIONAIS DE SAÚDE PÚBLICA DA COMUNIDADE DE PAÍSES DE LÍNGUA OFICIAL PORTUGUESA (RENSP-CPLP)}

O ano de 2019 também marcou a formação da Rensp-CPLP. Essa proposta de uma rede de formação em saúde pública no âmbito da CPLP já era um objetivo do Plano Estratégico de Cooperação em Saúde da Comunidade (Pecs-CPLP). Em reunião realizada em Lisboa em fevereiro de 20 I 9, representantes das instituições formadoras de Portugal, Brasil, Angola, Cabo-Verde, Guiné-Bissau e Moçambique, bem como de outros representantes de países da CPLP que participaram por videoconferência, fundaram a rede e elaboraram o seu primeiro Plano de Trabalho.

Desde então foi desenvolvido também um plano de comunicação, com o lançamento de um sítio web para a rede (Rensp/CPLP, 2020), e realizadas reuniões virtuais de organização. Em 2020, o Plano de Trabalho foi atualizado e integrado ao calendário 
do Pecs, com atividades previstas para os dois últimos anos do ciclo atual. Uma das primeiras atividades previstas foi a realização de diagnóstico das capacidades formativas (cursos, outras ações formativas e tecnologias educacionais) existentes nas instituiçõesmembros da rede por meio de um questionário de mapeamento. A Secretaria Executiva da Rensp é exercida conjuntamente pela Ensp/Fiocruz e pela Ensp Nova, da Universidade Nova de Lisboa, em Portugal.

\section{AS REDES DE INSTITUTOS NACIONAIS DE SAÚDE}

Os Institutos Nacionais de Saúde Pública (INSP) são organismos científico-técnicos especializados de Estado que executam funções cruciais em saúde pública, tais como a vigilância, detecção e monitoramento de doenças; investigação e controle de surtos; análise de informações para apoiar a formulação de políticas; pesquisa; formação de recursos humanos; promoção da saúde e ciências laboratoriais. Dado seu caráter científico, os INSP são fontes confiáveis de assessoramento para níveis políticos e de decisão, com uma missão de longo prazo vinculada ao alcance de decisões autônomas e soberanas, baseadas nas realidades próprias dos respectivos países e regiões.

Tendo como fundamento essas funções, cruciais para a estruturação de sistemas sólidos de saúde, a Associação Internacional de Institutos Nacionais de Saúde Pública (IANPHI, na sigla em inglês) foi criada em 2006, no Rio de Janeiro, por 39 membros fundadores, incluindo, entre outros, os institutos de Saúde Pública do Brasil (Fiocruz), da Finlândia (KTL), de Portugal (IHMT) e dos EUA (CDC), contando com um subsídio inicial de 20 milhões de USD da Fundação Bill \& Melinda Gates por intermédio do Instituto de Saúde Global da Universidade de Emory. Em fins de 2019, a IANPHI contava com II 4 instituições-membros em 99 países, cobrindo uma população de mais de 5 bilhões de pessoas.

A prioridade de IANPHI é fortalecer os sistemas nacionais de saúde mediante 0 estabelecimento e desenvolvimento de INSP. Contando com a colaboração dos seus institutos-membros, de outras instituições associadas e de plataformas de cooperação técnica entre pares, a IANPHI apoia países de escassos recursos na criação dos seus respectivos institutos.

Durante a epidemia, a IANPHI tem realizado eventos em rede virtual (webinars) especificamente direcionados aos seus institutos-membros. Nesses eventos foram apresentadas as experiências dos institutos nacionais de Saúde Pública da Itália, Coreia, China e África Ocidental no combate à Covid, além de temas estratégicos como a redução das medidas de lockdown na Europa, instrumentos para a predição da evolução da pandemia e a mobilização das ciências comportamentais durante a emergência. 
Nos últimos anos, a IANPHI promoveu a constituição de redes regionais com o objetivo de fortalecer a cooperação regional, facilitar o apoio mútuo e a assistência técnica e fortalecer as respectivas capacidades. A associação dispõe de quatro redes regionais, na África, Ásia, Europa e América Latina e Caribe, esta última particularmente ativa durante a pandemia.

A Fiocruz participa de duas redes internacionais de institutos nacionais de saúde pública e as coordena: a Rede de Institutos da Comunidade de Países de Língua Portuguesa (Rinsp-CPLP) e a Rede Regional Latino-Americana e do Caribe (Rede IANPHI LatAm / Rins-Unasur). Ambas foram criadas há aproximadamente uma década com o objetivo de fortalecer estruturas científicas e técnicas de Estado que reduzam a dependência externa e consolidem soluções soberanas para os problemas nacionais, regionais e internacionais de saúde, mediante o esforço solidário de pares.

\section{Rinsp-CPLP}

A Rede de Institutos da Comunidade de Países de Língua Portuguesa (Rinsp-CPLP) foi criada em 22 de março de 201 I na cidade de Bissau, com a presença de representantes dos institutos de Saúde Pública dos cinco Países Africanos de Língua Portuguesa, os Palop (Angola, Cabo Verde, Guiné-Bissau, Moçambique e São Tomé e Príncipe), mais os de Timor Leste, Brasil e três institutos de Portugal, de acordo com a resolução do Plano Estratégico de Cooperação em Saúde da Comunidade (Pecs/CPLP).

A rede está ativa desde então, concentrando esforços no apoio mútuo para a criação de institutos nos países-membros que não possuem essa estrutura e para a elaboração, implementação e avaliação dos respectivos planos estratégicos para o seu fortalecimento. Dessa forma, no período foram criados os institutos de Saúde Pública em Guiné-Bissau e Cabo Verde; foi fortalecido o Instituto Nacional de Saúde de Moçambique, que se tornou referência técnica e científica para o país e para toda a região; foi apoiado o planejamento do Instituto Nacional de Investigação em Saúde de Angola e foram desenvolvidas bases conceituais para a criação de institutos em São Tomé e Príncipe e em Timor Leste.

Com o início da epidemia de Covid-19, a programação de cooperação técnica previamente estabelecida com base nas diretrizes do Plano Estratégico de Cooperação em Saúde teve que ser suspensa. No seu lugar foi criado um ciclo de reuniões virtuais bissemanais entre os diretores das suas instituições-membros ou seus representantes.

Inicialmente, foi realizada uma consulta temática que atendesse a três objetivos principais: I) a realização de debates sobre assuntos de interesse prioritário relativos ao papel dos institutos no combate à epidemia; b) seleção de projetos em curso nos 
institutos que pudessem ser objeto de colaboração para a sua execução regional, com potencial para a captação de recursos financeiros externos; e c) ações específicas de cooperação técnica por meio virtual.

De acordo com essa agenda, foram realizados, até o momento da publicação deste trabalho, três encontros temáticos virtuais que trataram, respectivamente, do papel dos institutos nas questões relacionadas à pesquisa genômica do vírus e aos seus usos práticos; do estado da arte do conhecimento a respeito da imunidade em Covid- 19 e dos usos das provas sorológicas para o diagnóstico e o monitoramento epidemiológico; e do papel da atenção primária em saúde no combate à epidemia. Os mesmos temas foram debatidos no âmbito da Rede Regional da América Latina e Caribe da IANPHI. As principais conclusões, que têm impacto significativo nas estratégias de combate à Covid, serão analisadas mais adiante.

\section{Rins-Unasul e Rede IANPHI-LatAm}

Dependente do Conselho de Saúde Sul-Americano, também existia a Rede SulAmericana de Institutos Nacionais de Saúde Pública (Rins-Unasul), efetivada em 2010 sob a coordenação do Instituto Nacional de Saúde do Peru e com a Fiocruz atuando como a sua Secretaria Executiva.

O principal objetivo da Rins-Unasul foi cooperar mutuamente para a criação e consolidação dos institutos nacionais de Saúde de todos os seus países-membros, mantendo reuniões presenciais periódicas e realizando missões de cooperação técnica, principalmente no campo do planejamento estratégico.

Como resultado da ruptura, em 2018, da maioria dos governos da região com a Unasul, a sua rede de institutos deixou de operar como tal. Entretanto, continuou constituída a partir do final desse mesmo ano, quando a Associação Internacional de Institutos Nacionais de Saúde Pública (IANPHI) criou a Rede Regional da América Latina e Caribe sob a coordenação da Fiocruz, a rede IANPHI LatAm, que com o aparecimento da pandemia adquiriu maior presença analisando e debatendo importantes aspectos do papel que cabe aos institutos exercer no seu combate.

A referida rede é composta pelos institutos de Argentina, Bolívia, Brasil, Colômbia, Costa Rica, Chile, Equador, El Salvador, México, Panamá, Peru e Venezuela, além da Agência Caribenha de Saúde Pública (CARPHA). 


\section{As redes de institutos diante da pandemia da Covid-19}

A pandemia potencializou os encontros virtuais entre seus membros, que têm servido para discutir diversos temas priorizados por eles. Em ambas as redes as principais questões foram semelhantes.

Com relação ao uso da genômica, ficou evidenciado que o acesso a essa tecnologia está reduzido apenas aos países de maior desenvolvimento econômico, que conseguiram realizar mapas genômicos completos de diversas cepas circulantes nos seus países e em alguns dos outros países das suas respectivas redes. Por meio desse mecanismo foi possível identificar origens diversas da introdução e da distribuição interna do vírus e avaliar as potenciais mutações genéticas, que teriam reflexos evidentes na elaboração de vacinas e na predição de variações no curso de desenvolvimento da gravidade da doença.

A discussão sobre o uso das provas sorológicas evidenciou novamente a grande iniquidade entre os diversos países que compõem ambas as redes. É consenso entre todos os cientistas que essas provas só devem ser utilizadas para inquéritos populacionais, sendo desaconselhado o seu uso para o diagnóstico individual, mas alguns países às voltas com sérias dificuldades orçamentárias para acessar os testes moleculares aconselhados para tanto têm desenvolvido estratégias algorítmicas para utilizar os testes sorológicos como indicadores preliminares e os moleculares apenas para confirmação.

Com relação ao papel dos institutos na atenção primária para o combate à pandemia, lamentavelmente a resposta da grande maioria destes é bastante reduzida.

Para os próximos encontros estão propostos, entre outros, os temas: acesso às vacinas e outros medicamentos como bens públicos comuns; papel dos institutos na prevenção e controle dos desastres naturais; desigualdades.

\section{A REDE INTERNACIONAL DE EDUCAÇÃO DE TÉCNICOS EM SAÚDE (RETS)}

A Rede Internacional de Educação de Técnicos em Saúde (Rets) é uma articulação entre instituições e organizações envolvidas com a formação e a qualificação de pessoal técnico da área da saúde. Sua missão é fortalecer os sistemas nacionais de saúde, com base no pressuposto de que a qualificação dos trabalhadores é uma dimensão fundamental para a implementação de políticas públicas de saúde que atendam às necessidades das populações.

A Rets, criada em 1996 tendo como Secretaria Executiva a Escola de Saúde Pública da Costa Rica, funcionou até 200I, quando foi desativada. Em setembro de 2005, com a transferência da Secretaria Executiva para a Escola Politécnica de Saúde Joaquim 
Venâncio (EPSJV/Fiocruz), que havia se tornado Centro Colaborador da Opas/OMS para a Educação de Técnicos em Saúde em 2004, a rede foi reativada e hoje reúne 122 membros, de vinte países, entre instituições formadoras, órgãos de governo, organismos multilaterais e associações profissionais.

Ao fim de 1995, com coordenação da Opas/OMS, foi realizado amplo estudo sobre a situação da formação do pessoal técnico em saúde, que reuniu 70 centros em 16 países do continente americano. A pesquisa identificou inúmeros problemas, entre os quais sérias deficiências nos processos de planejamento de recursos humanos, inexistência de informação sistematizada sobre educação e trabalho desses profissionais e desatualização dos planos de estudo. A construção de uma rede internacional de educação de técnicos em saúde foi definida como prioritária e considerada importante forma de potencializar o conhecimento e a experiência acumulada, assim como de estimular o crescimento dos diferentes grupos, seja de docência, pesquisa ou prestação de serviços de saúde. São objetivos da rede:

I. possibilitar a cooperação técnica e a colaboração entre os membros na área da formação e desenvolvimento de pessoal técnico em saúde;

2. difundir informações resultantes de pesquisas sobre os trabalhadores desse nível a fim de fortalecer seu desenvolvimento, assim como dos serviços de saúde de que participam;

3. potencializar o acúmulo de conhecimento, estimulando a integração e o crescimento de grupos de docentes, pesquisadores, planejadores, administradores e prestadores de serviço;

4. promover o crescimento e o fortalecimento das suas instâncias organizativas e estimular a captação de recursos financeiros para garantir a sua sustentabilidade;

5. identificar as necessidades educacionais e de formação, bem como outros requisitos básicos das diferentes especialidades técnicas existentes.

\section{A Rets-CPLP e a Rets-Unasul: unindo esforços para fortalecer a formação de técnicos em saúde}

Em maio de 2009, a aprovação do Plano Estratégico de Cooperação em Saúde da CPLP foi o ponto de partida para a criação, em dezembro do mesmo ano, da Rede de Escolas Técnicas de Saúde da CPLP (Rets-CPLP). Na ocasião também foi criada a Rede de Escolas Técnicas da Unasul (Rets-Unasul), cuja origem está relacionada à criação do então Conselho de Saúde Sul-Americano e à Agenda Sul-Americana da Saúde, 
estabelecida em dezembro de 2008. Naquele momento, ambas as redes foram formadas como sub-redes da Rets, já amplamente consolidada.

Na 4 a Reunião Geral da Rets, realizada em novembro de 2018 no Rio de Janeiro, e com a extinção da Unasul, seus membros decidiram estabelecer, além do Plano de Trabalho e do Plano de Comunicação da rede, um Plano de Ações Regionalizadas para a América Latina. Na mesma ocasião também foi realizada a 4 a Reunião Ordinária da Rets-CPLP.

\section{A Rets e a pandemia de Covid-19: comunicação permanente entre os membros}

Na reunião de novembro de 2018, os planos de trabalho aprovados já previam o incremento do uso das tecnologias de informação e comunicação (TIC) para fortalecer o trabalho da rede. Nesse sentido, foram intensificadas tanto as ações de comunicação com o público externo, por meio do website da Rets (Rets, 2020a), de suas redes sociais (Facebook, Twitter, Instagram e YouTube) e de seu boletim eletrônico quinzenal, quanto a comunicação interna, entre os membros da rede, por meio do informe trimestral da Secretaria Executiva e de grupos de WhatsApp.

Essas iniciativas se mostraram essenciais no contexto da pandemia, pois acabaram proporcionando um incremento das ações que vinham sendo realizadas, mas também a possibilidade e a necessidade de pensar na manutenção da rede num cenário futuro de poucos recursos financeiros e possíveis dificuldades na realização de atividades presenciais.

A primeira ação definida foi a criação de um grupo de WhatsApp específico para discutir os aspectos da pandemia no âmbito da América Latina. A partir daí, foram realizadas a I ${ }^{a}$ Reunião Virtual da Rets para os países latino-americanos, em 22 de maio deste ano, com a presença de 45 pessoas (Rets, 2020b), e a I ${ }^{a}$ Reunião Virtual da RetsCPLP, em I de julho, com mais de 30 participantes (Rets, 2020b). Nos dois encontros, a prioridade foi discutir a formação de técnicos em saúde no contexto da pandemia. Nas reuniões, os países apresentaram os desafios que estão enfrentando e começaram a discutir novas possibilidades de cooperação e a continuidade de ações já em andamento.

De acordo com os membros, dois grandes problemas são as desigualdades sociais que impedem o acesso de grande número de alunos às tecnologias de ensino a distância e a impossibilidade de acesso aos serviços de saúde por alunos em fase de estágio. Tais problemas podem gerar enorme lacuna na formação dos técnicos em saúde, com reflexos bastante negativos nos serviços de atenção à saúde das populações. 
Das propostas aprovadas nas reuniões, é possível destacar:

1. A criação de uma página Covid-19 no website da Rets, para a divulgação de materiais produzidos sobre a Covid- 19 e compartilhados por seus membros.

2. A realização, em caráter virtual, do I Colóquio Virtual Latino-Americano de Educação Interprofissional e Formação de Técnicos em Saúde, cuja versão presencial seria realizada em maio de 2020, na Colômbia.

3. A publicação de uma edição especial Covid-19 da Revista Rets, em formato eletrônico, abordando inúmeros aspectos da pandemia e seus reflexos na formação e no trabalho dos técnicos em saúde.

4. A tradução emergencial de materiais didáticos e informativos sobre Covid-19 para atender um número maior de países. Nesse sentido foi lançada, em julho, a versão em espanhol da cartilha "Orientações para cuidadores domiciliares de pessoa idosa na epidemia do Coronavírus - Covid- I9" (Groisman et al., 2020) e está sendo discutida a possibilidade de tradução da "Ferramenta de bolso para agentes de saúde e cuidadores na ativa em defesa da vida na epidemia Covid- 19" (Leal et al., 2020) e do "Manual sobre biossegurança para reabertura de escolas no contexto da Covid-19" (Pereira et al., 2020), todos publicados pela EPSJV/Fiocruz.

5. A elaboração de um curso virtual, adequado às condições tecnológicas dos Palop, fruto de parceria entre a Escola Superior de Tecnologia da Saúde de Lisboa (ESTeSL) e a EPSJV/Fiocruz; e

6. O estabelecimento, via Coordenação da Rede Universitária de Telemedicina (Rute), de uma parceria com a Rede Nacional de Ensino e Pesquisa (RNP) para a distribuição de salas de webconferência para membros dos Palop que tenham interesse em utilizar a plataforma desta rede para reuniões e processos formativos durante a pandemia. Inicialmente foram disponibilizadas dez salas e, caso necessário, outras poderão sê-lo.

Além dessas atividades, a Rets tem buscado incrementar sua interação com outras redes e parceiros para a organização de eventos virtuais sobre inúmeros aspectos relacionados à pandemia, como foi o caso do webinar "As vacinas e medicamentos para a Covid- 9 como bens públicos globais“, realizado em 30 de julho (Cris/Fiocruz, 2020).

Também está prevista, ainda este ano, a realização de um webinar sobre os desafios das instituições para o retorno às atividades presenciais de formação. 


\section{REDE GLOBAL DE BANCOS DE LEITE HUMANO (rBLH-BR)}

A Rede Brasileira de Bancos de Leite Humano (rBLH-BR), cuja coordenação está sediada na Fiocruz com suporte técnico do Instituto Nacional de Saúde da Mulher, da Criança e do Adolescente Fernandes Figueira (IFF) e do Instituto de Comunicação e Informação Científica e Tecnológica em Saúde (lcict), conta com 224 bancos de leite humano distribuídos em todos os estados do território nacional. O modelo brasileiro é reconhecido mundialmente pelo desenvolvimento tecnológico inédito que alia baixo custo a alta qualidade. Em razão dessa experiência, a rBLH-BR coordena também a Rede Global de Bancos de Leite Humano, que tem o objetivo de, mediante cooperação técnica internacional, formar multiplicadores que possibilitem a transferência de tecnologia para outros países. A Rede Global apoia também organismos multilaterais como a SecretariaGeral Ibero-Americana, com a criação do Programa Ibero-Americano de Bancos de Leite Humano (2008), que integra todos os países da região da Ibero-América e a Comunidade de Países de Língua Portuguesa (2017).

Em tempos da pandemia de Covid-19, as discussões técnicas que a Rede Global de Leite Humano tem promovido em suas duas seções, a Ibero-Americana e a da CPLP, visam ao conjunto de políticas e iniciativas de enfrentamento voltadas para assegurar a segurança das doadoras, dos profissionais da saúde envolvidos em todas as etapas dos processos de trabalho dos BLHs e dos recém-nascidos contemplados pelas doações.

\section{A RBLH-CPLP no enfrentamento da Covid-19}

A Rede de Bancos de Leite Humano da Comunidade de Países de Língua Portuguesa (rBLH-CPLP) reuniu-se virtualmente em maio de 2020, em meio à pandemia de Covid-19, para apresentar os desafios enfrentados e as ações desenvolvidas para manter suas atividades. As recomendações de segurança em virtude da pandemia não interferem na continuidade da amamentação e extração do leite pela mãe, desde que de acordo com seu desejo, com suas condições clínicas e com as medidas recomendadas para evitar a transmissão do vírus. Algumas atitudes garantem a segurança, como lavar as mãos antes de tocar o bebê ou retirar o leite; usar e trocar adequadamente a máscara facial; e seguir todas as orientações e recomendações para a extração segura do leite.

A falta de informação e o grande desafio gerado pela Covid- 19 acabaram ampliando a atuação das redes em modalidades virtuais e a progressiva institucionalização que ajuda a criar novas oportunidades para abordar problemas comuns e articular respostas para a 
adoção de medidas coletivas, além de contribuir para o fortalecimento das capacidades institucionais para o enfrentamento dos problemas de saúde dos países.

O trabalho em rede pode ser uma opção significativa para cooperação técnica, troca de conhecimentos e experiências, treinamento, aprimoramento de capacidades e transferência de tecnologia em condições de mais igualdade de poder e recursos, bem como para aprendizagem mútua e coordenação de políticas e estratégias de desenvolvimento entre os países parceiros.

A pandemia tem permitido a adoção de modalidades de trabalho virtual com potencial para a cooperação em saúde que ainda podem ser exploradas em maior medida.

O aprendizado entre pares, como acontece no âmbito das redes, e a cooperação estruturante representam um enfoque poderoso e flexível para o fortalecimento de capacidades nacionais e para o empoderamento de todos os participantes em prol da saúde pública como um todo e, especificamente no momento atual, para o enfrentamento da pandemia da Covid- 19.

\section{REFERÊNCIAS}

ALMEIDA, C. M. et al. A concepção brasileira de "cooperação Sul-Sul estruturante" em saúde. Reciis Revista Eletrônica de Comunicação, Informação E Inovação em Saúde, 4(1): 25-35, 20 I0. Disponível em: <https://www.reciis.icict.fiocruz.br/index.php/reciis/article/view/696>. Aceso: 2/I0/20.

CENTRO DE RELAÇÕES INTERNACIONAIS EM SAÚDE (CRIS/FIOCRUZ). Vacinas e Medicamentos para Covid- 9 como Bens Públicos Globais. Seminário virtual latino-americano, 30 jul. 2020. Disponível em: $<$ https://www.youtube.com/watch?v=exjkp4BeSPo>. Acesso em: 18 set. 2020.

FRANCO, T. B. As redes na micropolítica do processo de trabalho em saúde. In: PINHEIRO, R.; FERLA, A. A. \& MATOS, R. A. Gestão em Redes: tecendo os fios da integralidade em saúde. Rio de Janeiro: Lappis, IMS/Uerj, Abrasco, 2006.

GOCAN, S. E LAPLANTE, M. A. Interprofessional collaboration in Ontario's family health teams: a review of the literature. Journal of Research in Interprofessional Practice and Education, 3(3): I - 19, 2014.

GROISMAN, D. et al. (Coords.). Orientações para Cuidadores Domiciliares de Pessoa Idosa na Epidemia do Coronavírus - Covid-19. Rio de Janeiro: EPSJV/Fiocruz, Icict/Fiocruz, 2020. Disponível em: < http:// www.epsjv.fiocruz.br/sites/default/files/files/CartilhaCuidadorldoso_Covid-19.pdf>. Acesso em: I8 set. 2020.

KICKBUSCH, I \& BERGER, C. Diplomacia da saúde global. Reciis - Revista Eletrônica de Comunicação, Informação E Inovação em Saúde, 4(1): 19-24, mar. 2010.

LEAL, A. L. G. et al. Ferramenta de Bolso para Agentes de Saúde e Cuidadores da Ativa em Defesa da Vida na Epidemia Covid-19. Rio de Janeiro: Disponível em: EPSJV/Fiocruz, 2020. Disponível em: < http://www.epsjv. fiocruz.br/sites/default/files/files/ferramenta\%20de\%20bolso\%203.pdf>. Acesso em: I 8 set. 2020. 
PEREIRA, I. D. F. et al. Manual sobre Biossegurança para Reabertura de Escolas no Contexto da Covid- 19. Rio de Janeiro: EPSJV/Fiocruz, 2020. Disponível em: < http://www.epsjv.fiocruz.br/sites/default/files/ manual_reabertura.pdf $>$. Acesso em: 18 set. 2020.

REDE DE ESCOLAS NACIONAIS DE SAÚDE PÚBLICA DA COMUNIDADE DOS PAÍSES DE LÍNGUA PORTUGUESA (RENSP/CPLP). Site. Disponível em: <http://rensp.ensp.fiocruz.br/>. Acesso em: 18 set. 2020.

REDE INTERNACIONAL DE EDUCAÇÃO DE TÉCNICOS DE SAÚDE (RETS). Site. Disponível em: < http:// www.rets.epsjv.fiocruz.br>. Acesso em: I 8 set. 2020a.

REDE INTERNACIONAL DE EDUCAÇÃO DE TÉCNICOS DE SAÚDE (RETS). O trabalho e a formação dos técnicos de saúde em tempos de pandemia de Covid-19. In: REUNIÃO VIRTUAL DA REDE DE ESCOLAS TÉCNICAS EM SAÚdE DA COMUNIDADE DOS PAÍSES DE LÍNGUA PORTUGUESA (RETS-CPLP), I, I 7 jul. 2020b, on-line. Disponível em: <http://www.rets.epsjv.fiocruz.br/I a-reuniao-virtual-da-retscplp>. Acesso em: 18 set. 2020.

REDE DE ESCOLAS E CENTROS FORMADORES EM SAÚDE PÚBLICA DA AMÉRICA LATINA (RESPAL). Declaração da Rede de Escolas e Centros Formadores em Saúde Pública da América Latina sobre a Pandemia de Covid-19, 2020. Disponível em: < http://www.respal.ensp.fiocruz.br/sites/default/files/ declaracao_covid-_resp-al.pdf>. Acesso em: 18 set. 2020.

ROVERE, M. Redes: hacia la construcción de redes en salud: los grupos humanos, las instituciones y la comunidad. Secretaría de Salud Pública. Rosario, 1998.

SOUZA, P. R. M. \& COSTA, P. P. Educação permanente em saúde na formação da Rede Brasileira de Escolas de Saúde Pública. Saúde em Debate, 43(n. esp.): I 16-129, 2019. Disponível em: <https://www. scielo.br/pdf/sdeb/v43nspel/0 I03-I I 04-sdeb-43-spe0 I-0I 16.pdf>. Acesso: I8 nov. 2019. 
Formato: $16 \times 23 \mathrm{~cm}$

Tipologia: GoudySnas Lt BT, GoudySans Md BT e Interstate-LightCondensed Rio de Janeiro, dezembro de 2020

Não encontrando nossos títulos em livrarias, contactar a Editora Fiocruz: Av. Brasil, 4036, térreo, sala II 2 - Manguinhos

21040-36I - Rio de Janeiro, RJ

Tel.: (2I) 3882-9039 e 3882-9007

Telefax: (2I) 3882-9006

editora@fiocruz.br

www.fiocruz.br/editora 
Passados os primeiros meses da pandemia do novo coronavírus no Brasil, o Observatório Covid- I 9 Fiocruz, em parceria com a Editora Fiocruz e com o apoio da Rede SciELO Livros, traz para o público leitor um conjunto de livros instantâneos sobre as análises nele realizadas desde que foi criado para subsidiar o seu combate.

Nesta série Informação para Ação na Covid-I 9 será apresentado um balanço do conjunto de documentos (notas e relatórios técnicos, boletins, informes, recomendações, ensaios, artigos, entre outros) produzidos em resposta à pandemia. Cada volume da série se estrutura em torno de um tema: aspectos globais da pandemia e da diplomacia em saúde; cenários epidemiológicos e vigilância em saúde; as políticas e a gestão dos serviços e sistemas de saúde; orientações para os cuidados e a saúde dos trabalhadores da saúde; impactos sociais e desigualdades sociais na pandemia.

Com a publicação destes estudos em livros de acesso aberto colocamos à disposição do público o conjunto de informações e conhecimentos gerados no âmbito do Observatório Covid- 19 Fiocruz, realizamos um balanço e uma reflexão sobre como chegamos ao cenário atual e apontamos caminhos para um futuro próximo. E, ao mesmo tempo, mantemos o registro histórico desse conhecimento produzido a quente, no calor da hora.

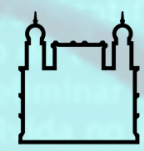

Ministério da Saúde 